\title{
Ethylenediamine Enhances Ionic Liquid Pretreatment Performance at High Solid Loading
}

$\mathrm{Li} \mathrm{Xu}^{\mathrm{a}, \mathrm{b}}$, Wen-Chao Li ${ }^{\mathrm{c}}$, Jiayu Xin ${ }^{\mathrm{d}}$, Sen-Jia Zhang ${ }^{\mathrm{c}}$, Bing-Zhi Li ${ }^{\mathrm{ab}, \mathrm{w}}$, and Ying-Jin Yuan ${ }^{\mathrm{a}, \mathrm{b}}$

a. Frontiers Science Center for Synthetic Biology and Key Laboratory of Systems Bioengineering (Ministry of Education), School of Chemical Engineering and Technology, Tianjin University, 92 Weijin Road, Nankai District, Tianjin 300072, China.

b. Collaborative Innovation Center of Chemical Science and Engineering (Tianjin), Tianjin University, 92 Weijin Road, Nankai District, Tianjin 300072, China.

c. Key Laboratory of Industrial Fermentation Microbiology (Ministry of Education), Tianjin University of Science and Technology, Tianjin 300457, China.

d. Beijing Key Laboratory of Ionic Liquids Clean Process, State Key Laboratory of Multiphase Complex System, Institute of Process Engineering, Chinese Academy of Sciences, Beijing 100190, China.

* Corresponding Author

E-mail: bzli@tju.edu.cn (Bing-Zhi Li)

Note:

The number of pages is 9 and the number of figures is 8 . 


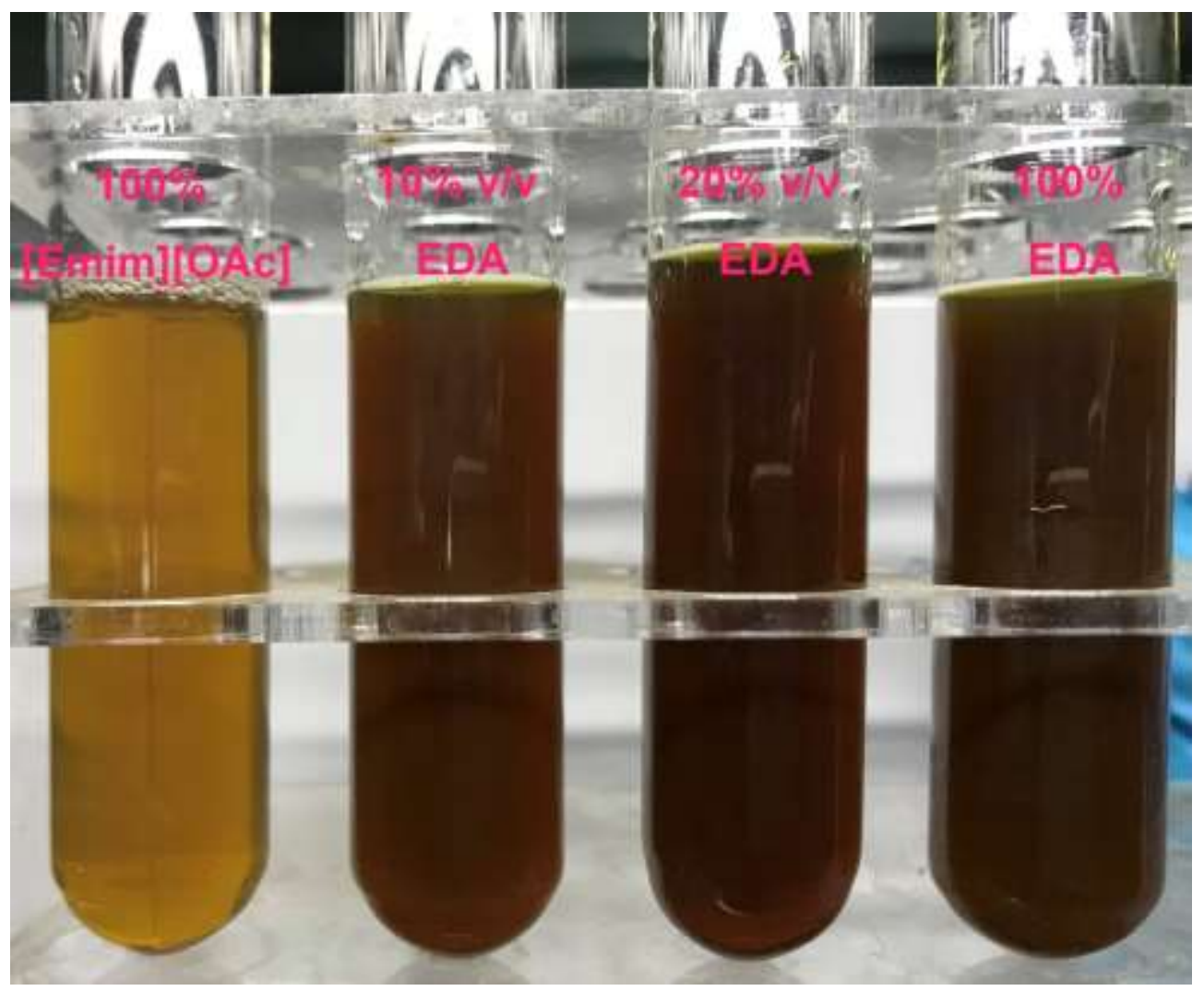

Figure S1. Wash liquids after pretreatment with different IL/EDA ratios. 
- Untreatd-G $\& 100 \% \mathrm{IL}-\mathrm{G} \vee 10 \% \mathrm{EDA}-\mathrm{G} \bullet 20 \% \mathrm{EDA}-\mathrm{G} \bullet 100 \% \mathrm{EDA}-\mathrm{G}$

ㅁ Untreatd-X $\triangle 100 \%$ IL-X $\nabla \quad 10 \%$ EDA-X $\odot 20 \%$ EDA-X $\odot 100 \%$ EDA-X

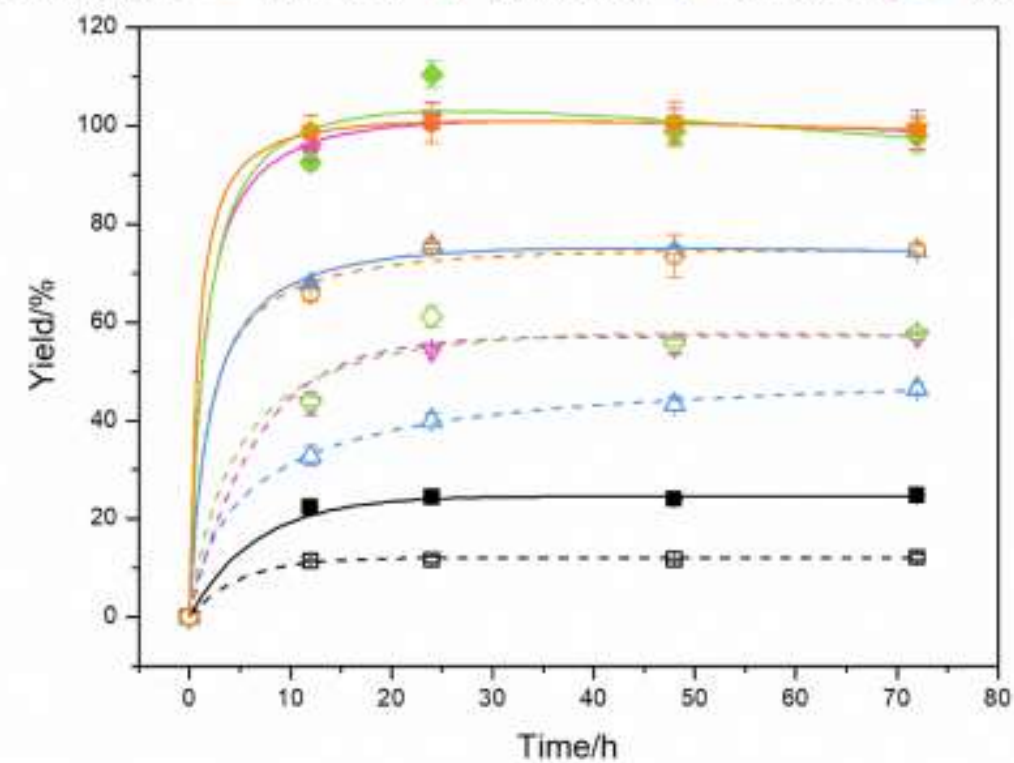

Figure S2. Sugar yields in the times of enzymatic hydrolysis of different pretreated CS. G: Glucan to glucose yields; X: Xylan to xylose yields. 


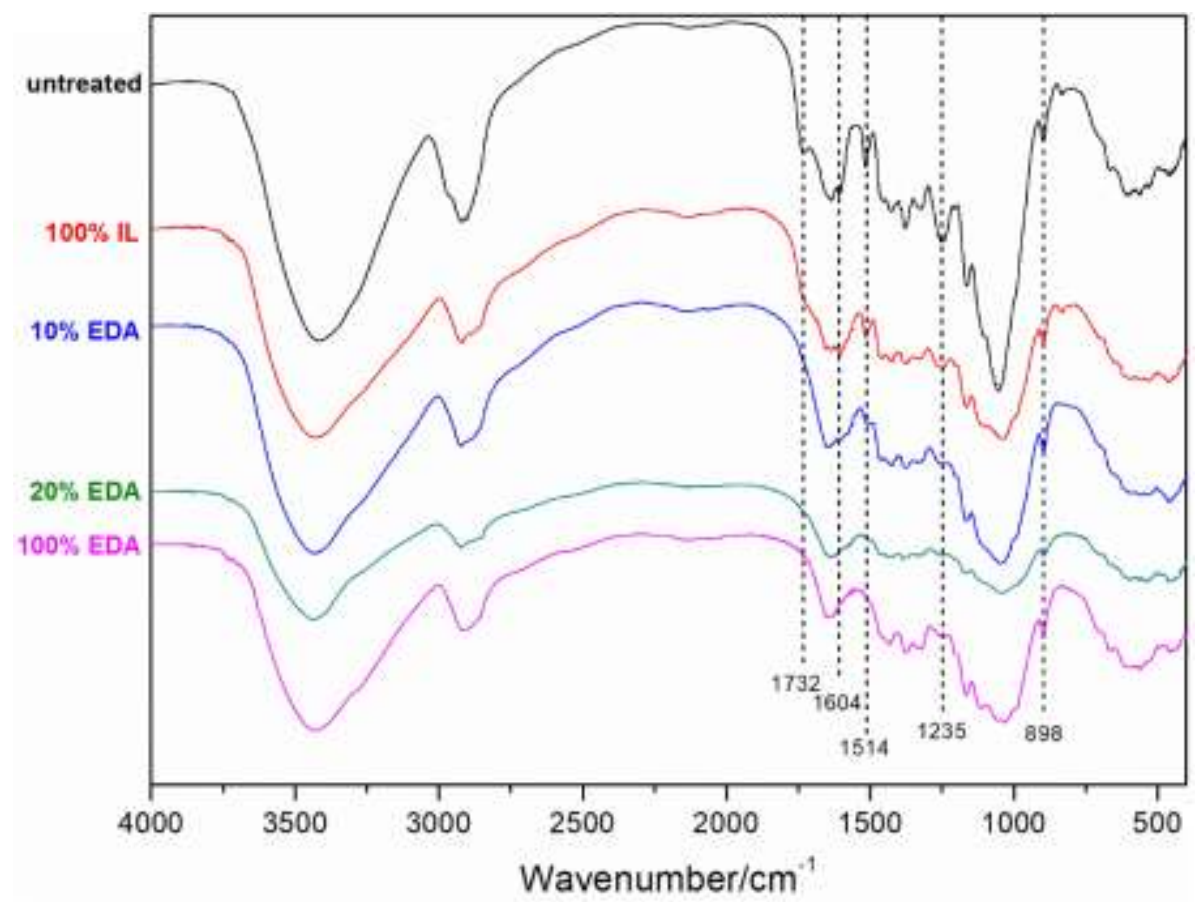

Figure S3. FTIR analysis of untreated CS and CS after different pretreatments. 

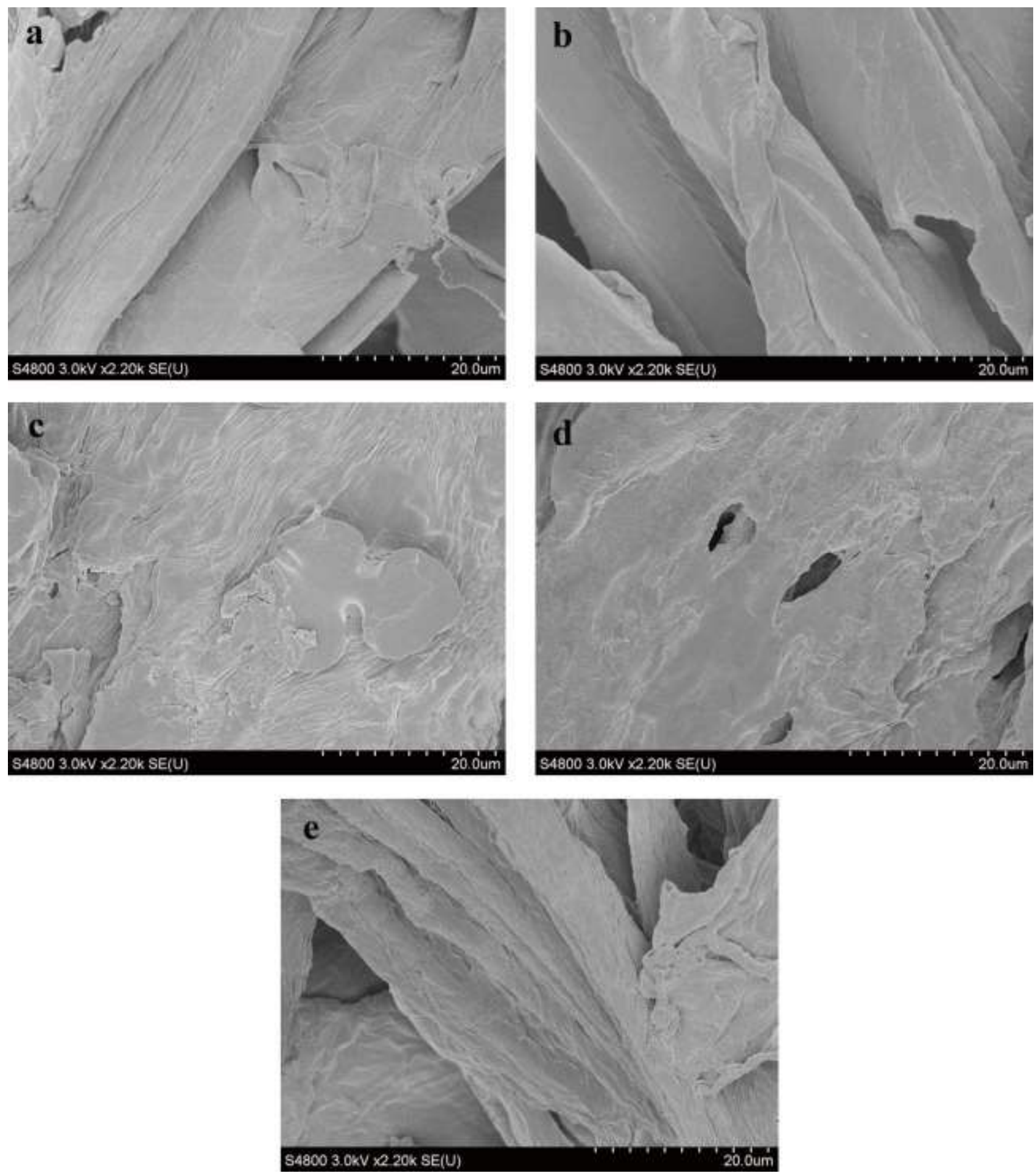

Figure S4. SEM images of untreated and pretreated CS. a: untreated CS; b: CS pretreated with 100\% IL; c: CS pretreated with $10 \%$ EDA; d: CS pretreated with 20\% EDA; e: CS pretreated with 100\% EDA. 

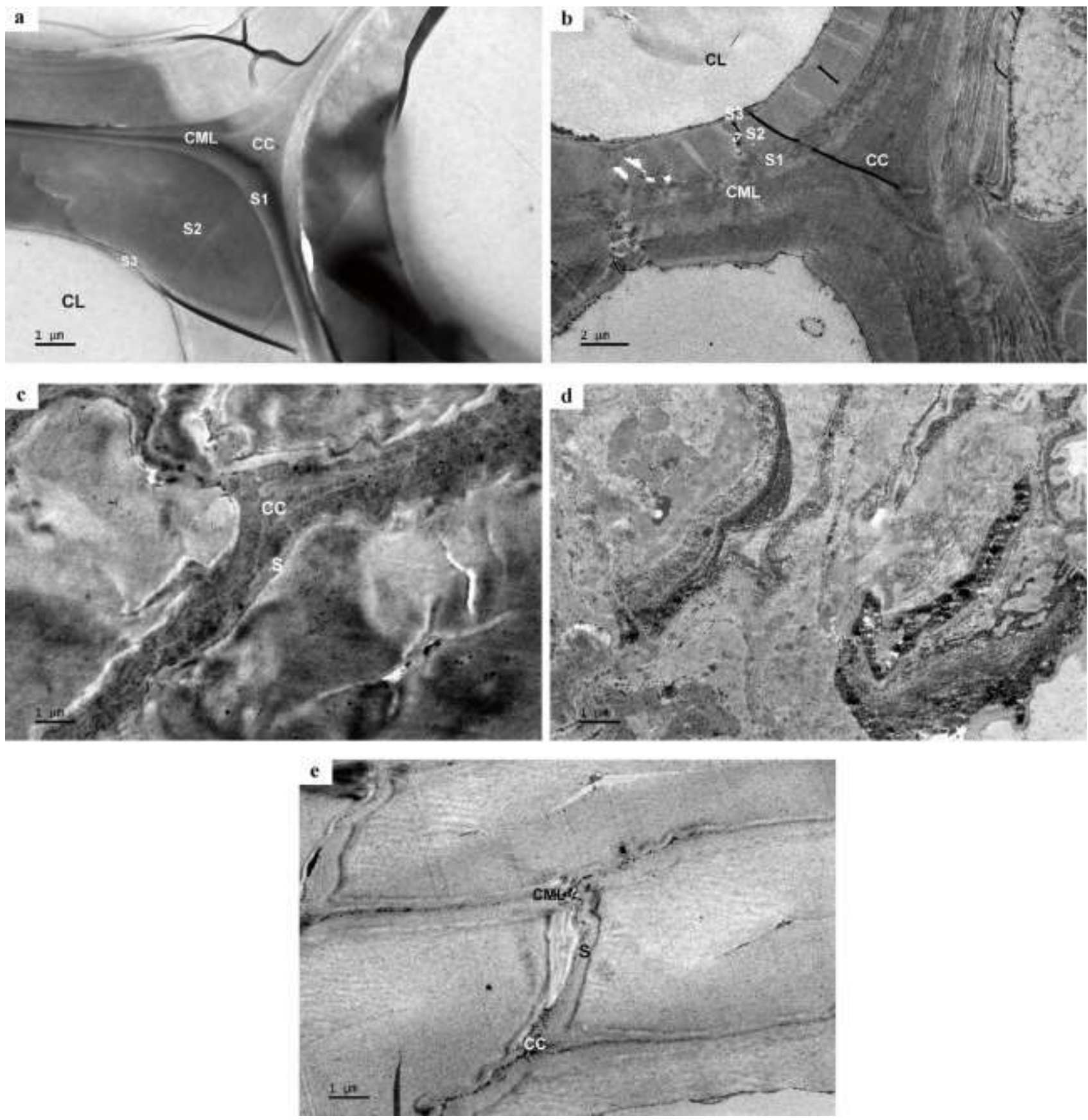

Figure S5. TEM images of untreated CS (a), CS pretreated with pure IL (b), 10\% EDA (c), 20\% EDA (d) and 100\% EDA (e). CC: cell corner; CML: compound middle lamella; S1: outer layer; S2: middle layer; S3: inner layer; CL: cell lumen. 


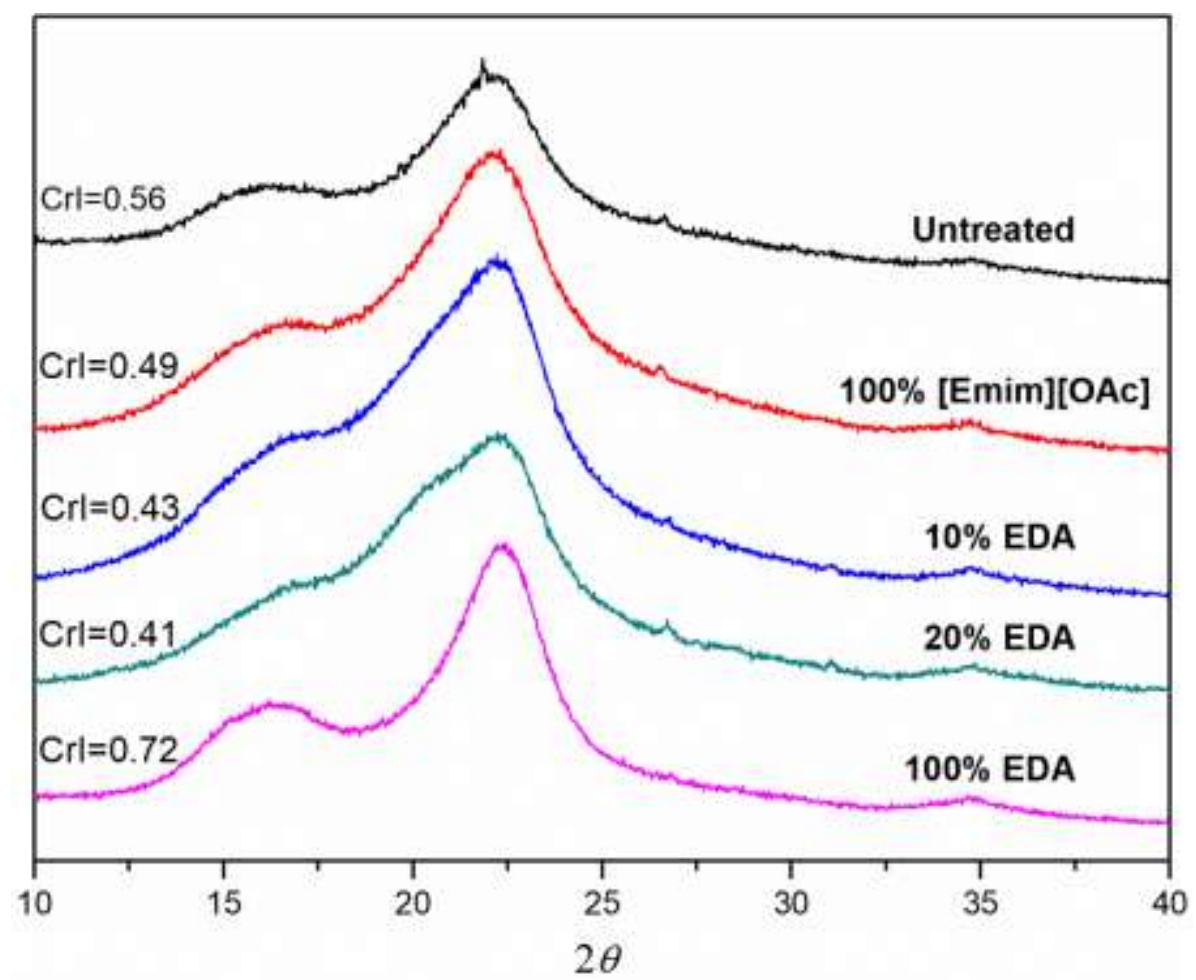

Figure S6. XRD diffraction profiles of untreated CS and CS after different pretreatment. 

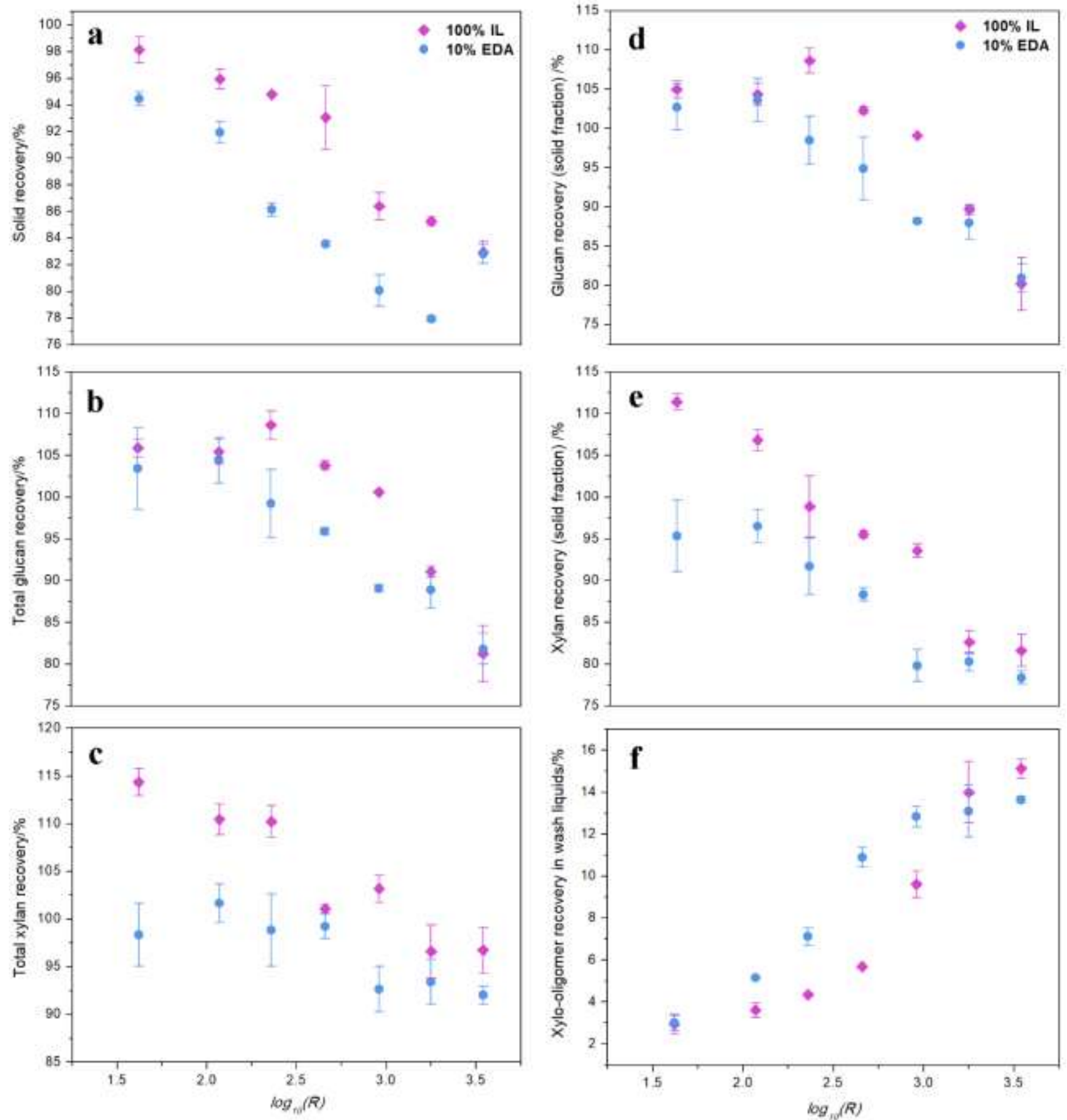

Figure S7. The effects of severity factor on solid recovery (a), total glucan recovery (b), total xylan recovery (c), glucan recovery (solid fraction) (d), xylan recovery (solid fraction) (e) and xylo-oligomer recovery in wash liquids (f). 

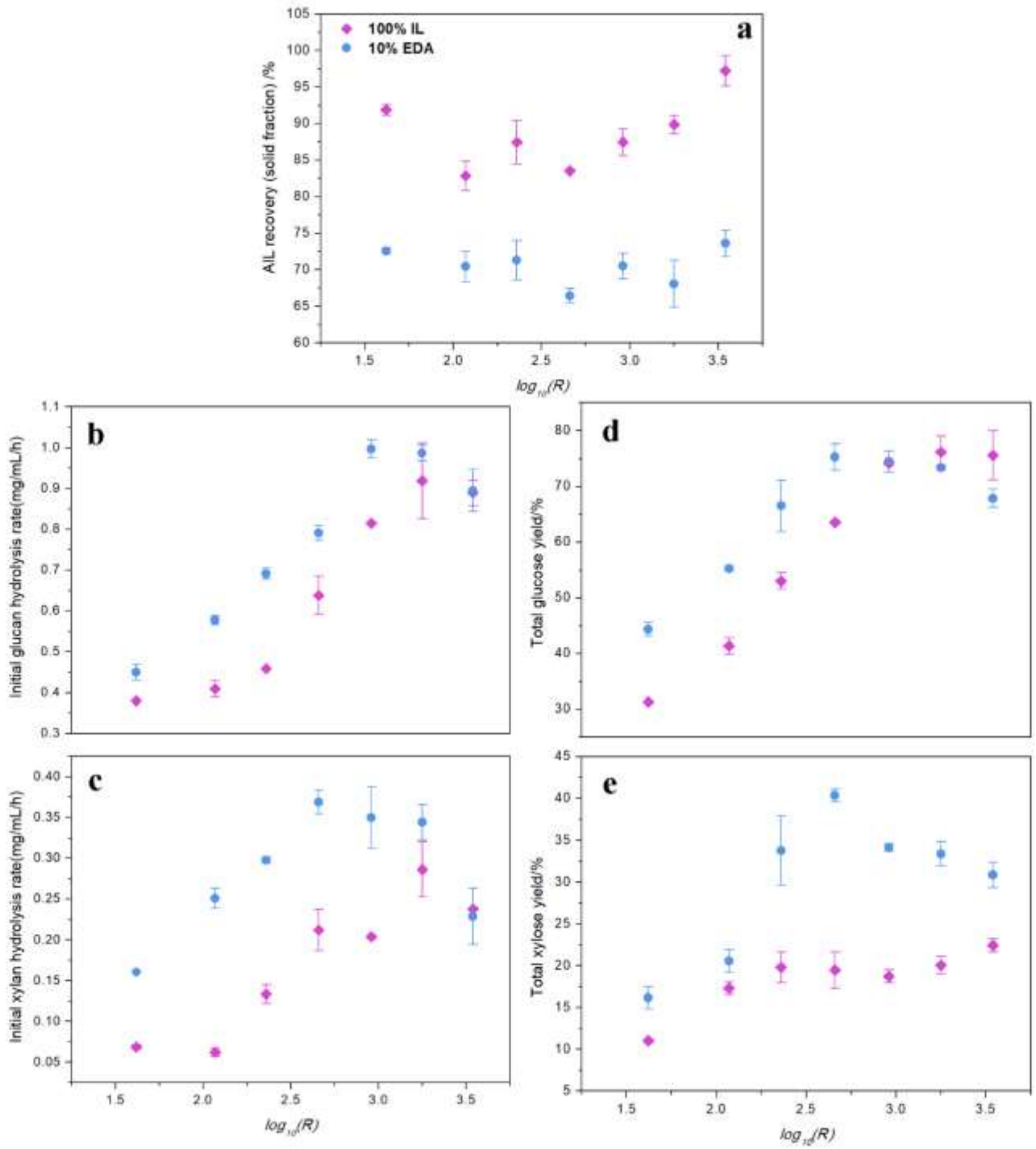

Figure S8. The effects of severity factor on AIL recovery (solid fraction) (a), initial glucan hydrolysis rate (b), initial xylan hydrolysis rate (c), total glucose yield $(72 \mathrm{~h}, \mathbf{d})$ and total xylose yield $(72 \mathrm{~h}, \mathbf{e})$. 